\title{
Criterion of stability of hydraulic cylinder and method of increasing its reliability under longitudinal-transverse loads
}

\author{
Dmitriy Kobzov ${ }^{1,}$, Sergei Repin ${ }^{2}$, and Vladimir Gubanov ${ }^{3}$ \\ ${ }^{1}$ Bratsk State University, Bratsk, 665709, Russia \\ ${ }^{2}$ Saint Petersburg State University of Architecture and Civil Engineering, St. Petersburg, 198005, \\ Russia \\ ${ }^{3}$ Science \&Technical Center «HydroTrans», St. Petersburg, 197374, Russia
}

\begin{abstract}
Currently, in most cases, the study of the operability of hydraulic cylinders in terms of their bearing capacity is carried out by means of assessing the longitudinal stability of a compressed rod with the variable cross section. In this case, the inclination of the rod in space is not taken into account. However, during operation, as a rule, a hydraulic cylinder inclined in space due to longitudinal-transverse loading is deformed in the vertical longitudinal plane with the appearance of a fullscale deflection. So, the limit value of the longitudinal compressive force is less than the limit compressive force of a stable rod, which implies that a rod having an initial curvature is more susceptible to bending, appearance of residual deformations in its elements and has less reliability than a straight, vertically arranged one. As the guide elements of the hydraulic cylinder wear out, its total deformation increases, which leads to an increase in longitudinal-transverse loads. With regard to long-stroke hydraulic cylinders, a special danger is their immediate stop in space when the working equipment of the machine meets an insurmountable obstacle. The purpose of the study is a scientifically based description of the areas of functioning of the hydraulic cylinder in the conditions of longitudinaltransverse bending or stability, as well as the boundaries separating them
\end{abstract}

\section{Introduction}

Multilink equipment of modern hydroficated road and construction machines (RCM) is driven by double-acting hydraulic cylinders with a one-sided rod, the operation of which is accompanied by their complex plane-parallel movement in space. At present, double-acting hydraulic cylinders with a one-sided rod are widely used in modern road and construction machines (RCM). Functionally, every hydraulic cylinder is exposed to a whole complex of operational longitudinal-transverse loads due to which it is deformed in the vertical longitudinal plane and a complete deflection appears, which sharply increases the acting bending loads and reactions in movable pressurized couplings. Currently, in most cases, the

* Corresponding author: kobzov7159@mail.ru 
study of the operability of hydraulic cylinders according to the parameters of their (load) bearing ability is carried out by means of assessing the longitudinal stability of a compressed rod with variable cross section. Moreover, according to its flexibility, a conclusion is drawn on the applicability of either Euler formula or Yasinsky formula, which, unfortunately, in no way take into account the inclination of the rod in space. However, during operation, as a rule, a hydraulic cylinder inclined in space due to longitudinal-transverse loading is deformed in the vertical longitudinal plane with the appearance of a complete deflection. In this case, the limit value of the longitudinal compressive force is less than the limit compressive force of a stable rod, which implies that a rod having an initial curvature is more susceptible to bending and the appearance of residual, plastic deformations in the sections of its elements and has less reliability than a straight, vertically arranged one. As the friction surfaces of the elements of the hydraulic cylinder wear out, which again leads to an increase in its total deformation and, respectively, to an increase in the acting longitudinal-transverse loads, the operating conditions of the hydraulic cylinder deteriorate with greater intensity, as a result of which its reliability and service life are reduced both in terms of its bearing and sealing abilities.

With regard to long-stroke hydraulic cylinders, their immediate stop in space is a particular danger when a multi-link equipment of a machine meets an insurmountable obstacle. All of the listed disadvantages of the traditional design of the hydraulic cylinder are eliminated by bringing the hydraulic cylinder from the state of longitudinal-transverse bending to the state of stability or close to that through the support of the body (liner) of the hydraulic cylinder with an intermediate support.

Hence, the aim of the work is a scientifically based description of the areas of functioning of the hydraulic cylinder under conditions of longitudinal-transverse bending or stability, the boundaries separating them, as well as developing recommendations for improving the design of the hydraulic cylinder. The object of the study is a hydraulic cylinder. The subject of the study is methods for assessing its bearing capacity in conditions of longitudinal-transverse bending and stability [1-8].

\section{Materials and Methods}

The methodology of the article includes the provisions of the mechanics of a deformed body, hydraulics, and technical operation.

Data processing was carried out using MathCAD and MathLab programs.

For the purpose of the article, the authors' works in the field of design and operation of hydraulic cylinders were used:

[Worley et al. (2006), Zimmerman et al. (2007), Dibir et al. (2008), Kobzov et al. (2010), Xia et al. (2013), Kobzov et al. (2016)].

\section{Results}

The hydraulic cylinder during operation is subject to longitudinal-transverse loading, leading to its longitudinal-transverse bending in the vertical longitudinal plane [1].

The longitudinal-transverse bending of an element should mean its deformation under the action of the longitudinal force. It happens due to the three main reasons: either there is an initial irregularity due to which the longitudinal force creates a bending moment relative to the center of gravity of the cross sections; or there is an eccentricity of the load application, i.e., the line of action of the longitudinal force does not pass through the center of gravity for solid sections or the center of bending for hollow ones; or there is a transverse 
load causing a deflection, which, in turn, creates a non-zero bending moment from the longitudinal force $P_{S}$.

In the case with RCM cylinders, especially long-stroke ones, all the three cases take place, as a result of which calculation is possible only according to the deformed scheme, that is, with full account for the total deflection of the hydraulic cylinder.

However, in a number of works, the calculation of compressed-curved rods during their longitudinal-transverse loading is recommended not to be carried out according to permissible stresses, but according to permissible loads. So, in [2], the limit value of the longitudinal compressive force $P_{S_{\mathrm{E}}}$ for the hydraulic cylinder and its allowable value are calculated by Euler formula.

$$
P_{S_{\mathrm{E}}}=\frac{\pi^{2} E I}{l^{2}}
$$

from the condition of loss of stability by a hydraulic cylinder or another similar element.

Where: $E$ is Young's modulus of the rod material, $I$ is the minimum moment of inertia of its section, and $l$ is the length of the rod.

It is clear that the rod with greater flexibility, when other parameters are unchanged, has lower compressive and bending compressive strength, due to which the depletion of the bearing strength for stability occurs, as a rule, before the material's safety factor has been exhausted.

However, when calculating longitudinal-transverse bending, it makes no sense to talk about the loss of stability in the plane of action of the transverse load, since, firstly, at any value of the axial force $P_{S}$, the rod experiences bending, and when it increases, there is no qualitative change in the nature of the deformation, as is the case with loss of stability. Secondly, Euler formula is applicable only under the condition

$$
\frac{\mu l}{i_{\min }}=\lambda \geq \lambda_{\lim }=\pi \sqrt{\frac{E}{\sigma_{\mathrm{pr}}}},
$$

where: $\lambda$ is the flexibility of the considered rod, depending on its reduced length $\mu l$, dimensions and shape of the cross section, that is, on the main central radius of inertia $i$, which is usually minimal $i_{\text {min }} ; l$ is the length of the rod; $\lambda_{\text {lim }}$ is limit or boundary flexibility for the material of the rod, depending only on the physico-mechanical properties: Young's modulus $E$ and the limit of proportionality $\sigma_{\mathrm{pr}}$ of the material of the rod; $\mu$ is the effective length factor.

Hence, the task of describing the boundary conditions and areas of functioning of the hydraulic cylinder, in which it may lose its working capacity due to loss of bearing capacity either because of limit longitudinal-transverse deformations, or because of the loss of stability, is of interest.

Currently, mechanical engineering aims to create hydraulic cylinders with parameters for the main and additional series in units of measurement according to standards GOST 6540-68, ST SEV 3936-82, GOST 16514-96, as well as ISO 2944, ISO 3320, ISO 3322 and ISO 4393 with the nominal pressure $\mathrm{p}=(0.63 \ldots 63) \mathrm{MPa}$, with piston (rod) stroke $z=(4 \ldots$ $10000) \mathrm{mm}$, with piston (liner) diameters $D_{1,3}=(4 \ldots 900) \mathrm{mm}$ and rod (sleeve) $D_{2,4}=(4 \ldots$ 900) $\mathrm{mm}$, with the ratio of the pressure areas in the piston and rod cavities $\varphi=(1.06 \ldots$ 5.26).

In relation to RCM hydraulic cylinders, these parameters lie in the ranges: $p=\left(\begin{array}{l}6 \ldots 25\end{array}\right)$ 
$\mathrm{MPa} ; z=(160 \ldots 2000) \mathrm{mm} ; D_{1,3}=(80 \ldots 180) \mathrm{mm} ; D_{2,4}=(55 \ldots 125) \mathrm{mm} ; \varphi=(1.33$ and 1.6).

The speed of movement of the rod in these documents is not specified, but lies in the range of $d z / d t=(0.1 \ldots 1.0) \mathrm{m} / \mathrm{s}$, and, as applied to RCM, does not exceed $0.5 \mathrm{~m} / \mathrm{s}$.

This paper considers one of the most common hydraulic cylinders with the following characteristics: $p=25 \mathrm{MPa} ; z=1400 \mathrm{~mm} ; D_{1,3}=140 \mathrm{~mm} ; D_{2,4}=90 \mathrm{~mm}$.

The hydraulic cylinders of multi-link RCM during the operation of the machine make significant spatial plane-parallel movements, which are described by the possible and working ranges of their movements [3, 4].

In accordance with the design schemes of the equipment of some multi-link RCMs proposed in [3, 4], as well as their geometric characteristics and parameters of the hydraulic cylinders used in them $[1,3]$, possible ranges of the spatial arrangement of the hydraulic cylinders as applied to the boom, stick and bucket hydraulic cylinders, for example, of single-bucket excavators EO-3322A and EO-4121A with a backhoe, are $88^{\circ}, 103^{\circ}, 214^{\circ}$ and $90^{\circ}, 105^{\circ}, 191^{\circ}$, respectively [3-5]. Moreover, the ranges of the spatial arrangement of these hydraulic cylinders only due to the movement of their own rods make up $88^{\circ}, 9^{\circ}, 7^{\circ}$ and $90^{\circ}, 6^{\circ}, 9^{\circ}$, respectively [5].

The working ranges of the spatial arrangement of the boom, stick and bucket hydraulic cylinders of a single-bucket excavator, in our case EO-3322A (backhoe), are $+84^{\circ} \ldots+19^{\circ}$, $-19^{\circ} \ldots-36^{\circ}$ and $-64 \ldots-111^{\circ}$, respectively [5].

Taking into account the provisions of [1], the compressive stresses $\sigma_{\text {comp }}\left(x_{\sigma}\right)$ arising in a dangerous cross section $x_{\sigma}$ of the hydraulic cylinder rod under longitudinal-transverse loading without considering the kinematic features of the working equipment of a specific RCM are analytically described by the equation:

$$
\sigma_{\text {comp }}\left(x_{\sigma}\right)=\frac{P_{S}}{F\left(x_{\sigma}\right)}+\frac{M_{Q}\left(x_{\sigma}\right)}{W\left(x_{\sigma}\right)}+\frac{P_{S} \cdot e\left(x_{\sigma}\right)}{W\left(x_{\sigma}\right)}+\frac{P_{S} \cdot y_{T}\left(x_{\sigma}\right)}{W\left(x_{\sigma}\right)} .
$$

Where: the first summand gives the value of the normal stress from the action of the longitudinal compressive force $P_{S}$, the second provides the value of the greatest compression stresses caused by the transverse load $M_{Q}\left(x_{\sigma}\right)$ from the weight of the hydraulic cylinder, the third and fourth ones are the same, caused by additional bending in the presence of eccentricity $e\left(x_{\sigma}\right)$ in its supports and total deflection $y_{T}\left(x_{\sigma}\right)$, which are the shoulder of the application of longitudinal compressive force $P_{S} ; F\left(x_{\sigma}\right)$ and $W\left(x_{\sigma}\right)$ are the area and moment of resistance of the rod section, respectively; $x_{\sigma}$ is the coordinate of its dangerous cross section.

The total deflection $y_{T}(x)$ of the hydraulic cylinder is equal to the sum of its components

$$
y_{T}\left(x_{\sigma}\right)=y_{\alpha}\left(x_{\sigma}\right)+y_{\beta}\left(x_{\sigma}\right)+y_{\gamma}\left(x_{\sigma}\right)+y_{\delta}\left(x_{\sigma}\right)+y_{P}\left(x_{\sigma}\right)+y_{Q}\left(x_{\sigma}\right) .
$$

At the initial moment of operation, the deflection $y_{T}(x)$ can be represented by the sum

$$
y_{T}\left(x_{\sigma}\right)=y_{T 0}\left(x_{\sigma}\right)+y_{P}\left(x_{\sigma}\right) .
$$

In equations (3) and (4) $y_{T}\left(x_{\sigma}\right)$ is complete deflection of the hydraulic cylinder; $y_{\alpha}\left(x_{\sigma}\right)$ is its deflection due to the selection of technological gaps in its movable couplings; $y_{\beta}\left(x_{\sigma}\right)$ is the same as a result of a possible initial (technological) curvature of its lengthy elements during manufacture; $y_{\gamma}\left(x_{\sigma}\right)$ is the same due to the operational curvature of its 
rod; $y_{\delta}\left(x_{\sigma}\right)$ is the same due to the selection of additional clearances as a result of radial deformation under the pressure of its body; $y_{P}\left(x_{\sigma}\right)$ is the same due to its longitudinal loading in the presence of the aforementioned strained and unstrained deformations; $y_{Q}\left(x_{\sigma}\right)$ is the same due to the transverse loading of the hydraulic cylinder from the action of the weights of its elements; $y_{T 0}\left(x_{\sigma}\right)$ is the same before the supply of the working fluid under pressure in the cavity of the hydraulic cylinder [1].

Replacing the stresses $\sigma_{\text {srt }}\left(x_{\sigma}\right)$ with the limit $\sigma_{\mathrm{ls}}$ and, transforming the equation (3) with respect to the longitudinal compressive force, we obtain the equation for finding its maximum value $P_{S_{\mathrm{B}}}^{\text {hor }}$ at which a horizontally or inclined hydraulic cylinder loses its working capacity under longitudinal-transverse loading as a result of limit deformation of its rod, in this case, a circular solid section (Fig. 1)

$$
P_{S_{\mathrm{bend}}}^{\mathrm{hor}}=\frac{\sigma_{1 \mathrm{~s}} W\left(x_{\sigma}\right)-M_{Q}\left(x_{\sigma}\right)}{0,125 D_{2}+\left[e\left(x_{\sigma}\right)+y_{T}\left(x_{\sigma}\right)\right]} .
$$

Since record (6) is valid for a horizontal and inclined hydraulic cylinders, then for a vertical one it transforms into (7)

$$
P_{S_{\text {bend }}}^{\text {vert }}=\frac{\pi D_{2}^{2}}{4} \sigma_{1 \mathrm{~s}}
$$

due to the fact that the characteristics $M_{Q}\left(x_{\sigma}\right)$ and $\left[e\left(x_{\sigma}\right)+y_{T}\left(x_{\sigma}\right)\right]$ included in equation (6) with this arrangement are equal to zero.

Taking into account the parameters $\left(x_{\sigma}, z^{k}, \Theta^{k}, p^{k}\right)$ [4] of the highest operational loading of a particular hydraulic cylinder of the equipment of hydroficated RCM, equation (6) transforms into

$$
P_{S_{\text {bend }}}^{\text {hor }}=\frac{0,1 \sigma_{1 \mathrm{~s}} D_{2}^{3}-M_{Q}\left(x_{\sigma}, z^{k}, \Theta^{k}, p^{k}\right)}{0,125 D_{2}+\left[e\left(x_{\sigma}, z^{k}, \Theta^{k}, p^{k}\right)+y_{T}\left(x_{\sigma}, z^{k}, \Theta^{k}, p^{k}\right)\right]} .
$$

Where, $\Theta^{k}$ is the angle of inclination of the hydraulic cylinder to the surface of gravity.

In this case, record (7) does not change regardless of the spatial location of the hydraulic cylinder.

The maximum allowable value of the longitudinal compressive force $P_{S_{\text {stab }}}$, provided that the hydraulic cylinder loses its working capacity due to its stability loss according to recommendation [6], is found from equation (9)

$$
\frac{64 P_{S_{\text {stab }}}}{\pi E_{1}\left(D_{5}^{4}-D_{3}^{4}\right) \operatorname{tg}\left[l_{\text {lin }} \frac{64 P_{S_{\text {stab }}}}{\pi E_{1}\left(D_{5}^{4}-D_{3}^{4}\right)}\right]}+\frac{64 P_{\text {stab }}}{\pi E_{2} D_{2}^{4} \operatorname{tg}\left(l_{\text {rod }} \frac{64 P_{S_{\text {stab }}}}{\pi E_{2} D_{2}^{4}}\right.}=0
$$


where $D_{5}$ is the outer diameter of the liner (body) of the hydraulic cylinder; $E_{1}$ and $E_{2}$ are Young's moduli of the liner and rod materials, respectively; $l_{\text {lin }}$ and $l_{\text {rod }}$ are the length of the liner and the outer part of the rod, respectively.

The limit compressive load $P_{S_{\text {stab }}}$ is related to the force $P_{S_{\mathrm{E}}}$ described by the wellknown Euler formula that takes into account the parameter $z^{k}$ of the highest operational loading of a particular hydraulic cylinder

$$
P_{S_{\mathrm{E}}}=\frac{\pi^{2} E_{1} I_{1}}{l_{\mathrm{hc}}^{2}\left(z^{k}\right)}=\frac{\pi^{3} E_{1}\left(D_{5}^{4}-D_{3}^{4}\right)}{64\left[l_{\text {lin }}+l_{\text {rod }}\left(z^{k}\right)\right]^{2}},
$$

the coefficient $\xi<1$

$$
P_{S_{\text {stab }}}=\xi P_{S_{\mathrm{E}}}
$$

considering the variability in length of the cross section of the hydraulic cylinder and being equal to $0.405,0.441$ and 0.297 , respectively, for boom, stick and bucket hydraulic cylinders of a single-bucket excavator of size IV with a specific value of effort $P_{S_{\mathrm{E}}}$ for each of them.

In equation (10): $I_{1}$ is the axial moment of inertia of the cross section of the liner equals in our case to $\frac{\pi\left(D_{5}^{4}-D_{3}^{4}\right)}{64}$, and $l_{\mathrm{hz}}\left(z^{k}\right)=\left[l_{\text {lin }}+l_{\text {rod }}\left(z^{k}\right)\right]$ is the total length of the hydraulic cylinder with the rod extended from the liner by $z^{k}$.

Based on the above, the maximum allowable longitudinal compressive force $P_{S}$, acting on the hydraulic cylinder without disruption due to excessive bending or loss of stability, should be defined as the minimum force $P_{S_{\min }}$ of the complex of forces $\left[P_{S_{\text {bend }}}^{\text {hor }}\right]$, $\left[P_{S_{\text {bend }}}^{\text {vert }}\right]$ and $\left[P_{S_{\text {stab }}}\right]$ taking into account the parameters $\left(x_{\sigma}, z^{k}, \Theta^{k}, p^{k}\right)$ of the highest operational loading of a particular hydraulic cylinder of the hydroformed RCM, then

$$
P_{S} \leq P_{S_{\text {min }}} \equiv\left\{\begin{array}{l}
{\left[P_{S_{\text {bend }}^{\text {hor }}}\right]=\frac{P_{S_{\text {bend }}^{\text {hor }}}}{k_{\mathrm{p}} k_{S}}} \\
{\left[P_{S_{\text {bend }}^{\text {vert }}}\right]=\frac{P_{S_{\text {bend }}^{\text {vert }}}}{k_{\mathrm{p}} k_{S}}} \\
{\left[P_{S_{\text {stab }}}\right]=\frac{P_{S_{\text {stab }}}}{k_{\mathrm{p}} k_{\text {st }}} .}
\end{array}\right.
$$

In (12): $k_{\mathrm{p}}$ is the coefficient that takes into account a possible increase in pressure in the hydraulic system of the RCM, $k_{\mathrm{p}}=1.15 ; k_{\mathrm{st}}$ is the margin of stability, for steel cylinders $k_{\mathrm{st}}$ $=(1.5 \ldots 3.0) ; k_{S}$ is the statistical safety factor.

However, Euler formula is applicable only under condition (2) [2], which imposes a restriction on equations (11) and (12).

Considering that the length coefficient $\mu$, which determines the nature of the fastening of supports, for most common in RCM double-acting hydraulic cylinders with a single- 
sided rod and eyelets on both sides, is equal to 1, the condition for the applicability of Euler formula (2) with respect to the minimum length of the hydraulic cylinder $l_{\mathrm{hz} m i n}$ for our example is as follows:

$$
l_{\mathrm{hz}}\left(z^{k}\right) \geq l_{\mathrm{hz}_{\min }}=\frac{\pi}{4} \sqrt{\frac{E_{1}}{\sigma_{\mathrm{pl}}} \frac{\left(D_{5}^{4}-D_{3}^{4}\right)}{\left(D_{5}^{2}-D_{3}^{2}\right)}}
$$

for the main central radius of inertia $i=\frac{1}{4} \sqrt{\frac{\left(D_{5}^{4}-D_{3}^{4}\right)}{\left(D_{5}^{2}-D_{3}^{2}\right)}}$.

For our example $\left(D_{3}=0.14 \mathrm{~m}, D_{5}=0.18 \mathrm{~m}, z=1.4 \mathrm{~m}\right)$, the limit flexibility $\lambda_{\text {lim }}$ according to the right side of equation (2) is 50.563, and the minimum allowable length of the hydraulic cylinder $l_{\mathrm{hzmin}}$ is $2.88 \mathrm{~m}$ for characteristics of the material of the liner $E_{1}=2.15 \cdot 10^{5} \mathrm{MPa}$ and $\sigma_{\mathrm{pl}}=830 \mathrm{MPa}$, for which $30 \mathrm{HGSA}$ steel was adopted. In our case $l_{\mathrm{hz}}\left(z^{k}\right)=3.45 \mathrm{~m}$.

Figure 2 shows the relationship between the minimum permissible length of the hydraulic cylinder $l_{\mathrm{hzmin}}$ and the inner diameter of its liner $D_{3}$. Below this line, Euler formula, according to (13), is not applicable, and any rod, due to its low flexibility because of its relatively small length and significant diameter, must be assumed to be stable.

Figure 3 shows the dependences of the flexibility $\lambda$ of the hydraulic cylinder on its full length $l_{\mathrm{hz}}$ for various values of the internal diameter of the liner $D_{3}$ according to GOST 6540-68 "Hydraulic cylinders and pneumatic cylinders. Main sizes", as applied to RCM, taking into account their limit flexibility $\lambda_{\text {lim }}$.

Given this information (Figs. 2 and 3), special attention from the standpoint of the hydraulic cylinder losing operability due to its loss of stability should be given to the rods of significant lengths with small diametrical dimensions.

Figure 4 shows the dependence of the force $P_{S_{\text {stab }}}$ on the total length of the hydraulic cylinder $l_{\mathrm{hz}}$ for various values of the inner diameter of the liner $D_{3}=80 \ldots 250 \mathrm{~mm}$, the nature of which confirms the correctness of the above recommendation.

In addition, it should be borne in mind that during operation, the hydraulic cylinder, as a result of applying $p=16 \ldots 20 \ldots 25 \mathrm{MPa}$ of the working fluid to its piston cavity, develops a pushing force $P_{S_{\mathrm{hz}}}$ on the rod, which reactively compresses the hydraulic cylinder.

In this case, the studies show (Figs. 5 and 6) that the force $P_{S_{\mathrm{hz}}}$ can exceed the force $P_{S_{\text {stab }}}$, that is, the hydraulic cylinder may lose its working capacity due to the loss of stability.

$$
\frac{\pi D_{3}^{2}}{4} p=P_{S_{\mathrm{hz}}} \geq P_{S_{\mathrm{stab}}}=\xi \frac{\pi^{3} E_{1}\left(D_{5}^{4}-D_{3}^{4}\right)}{64 l_{\mathrm{hz}}^{2}}
$$




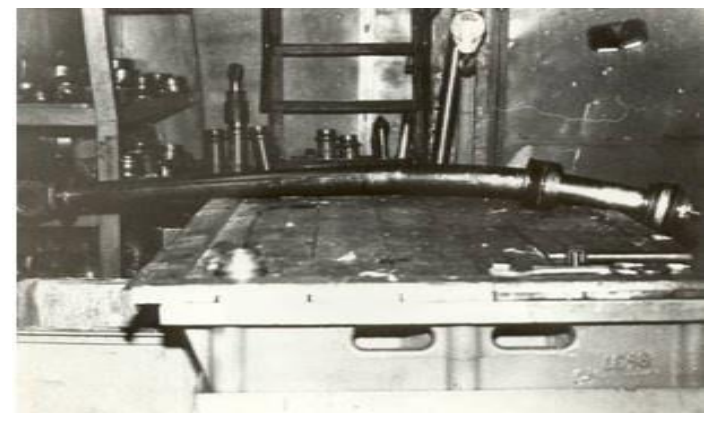

Fig.1. Rod bending D2 $=90 \mathrm{~mm}$ of the hydraulic cylinder of the single-bucket construction excavator, size IV.

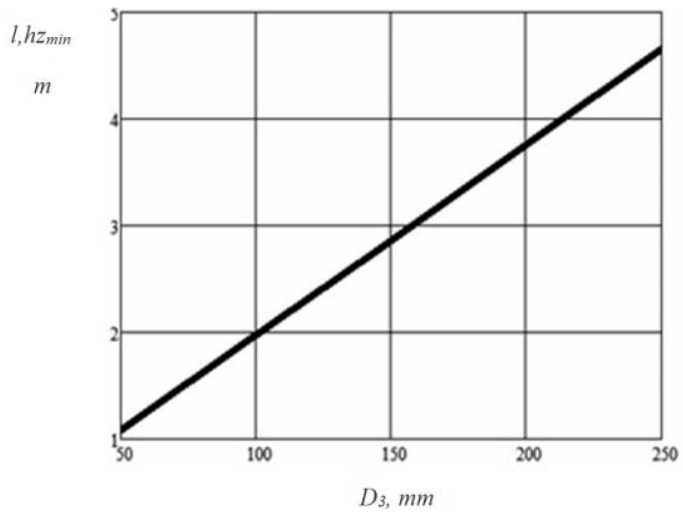

Fig. 2. The minimum permissible length of the hydraulic cylinder $l_{\text {hzmin }}$ for the inner diameter of the liner D3.

$\lambda$

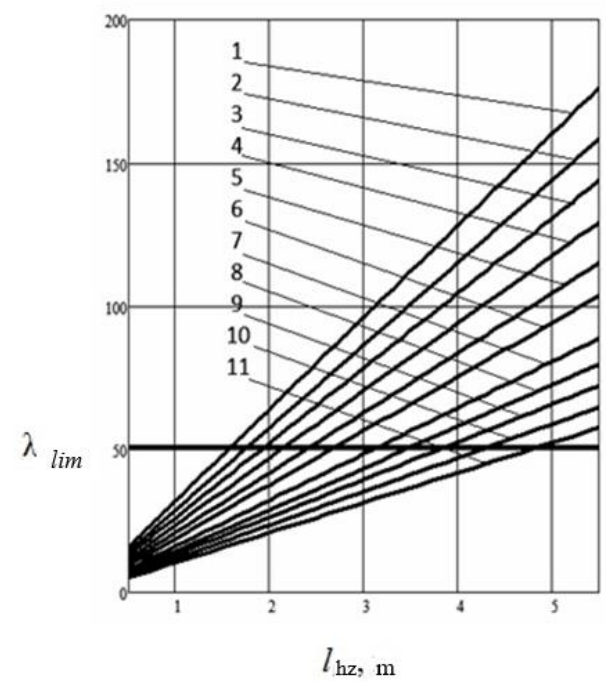

$D_{3}$

mm

80

90

100

110

125

140

160

180

200

220

250

Fig. 3. The dependences of flexibility $\lambda$ of the hydraulic cylinder (straight lines $1 \ldots 11$ ) from its full length $\mathrm{hz}$ for various values of the inner diameter of the liner D3 $=80 \ldots 250 \mathrm{~mm}$, respectively. 


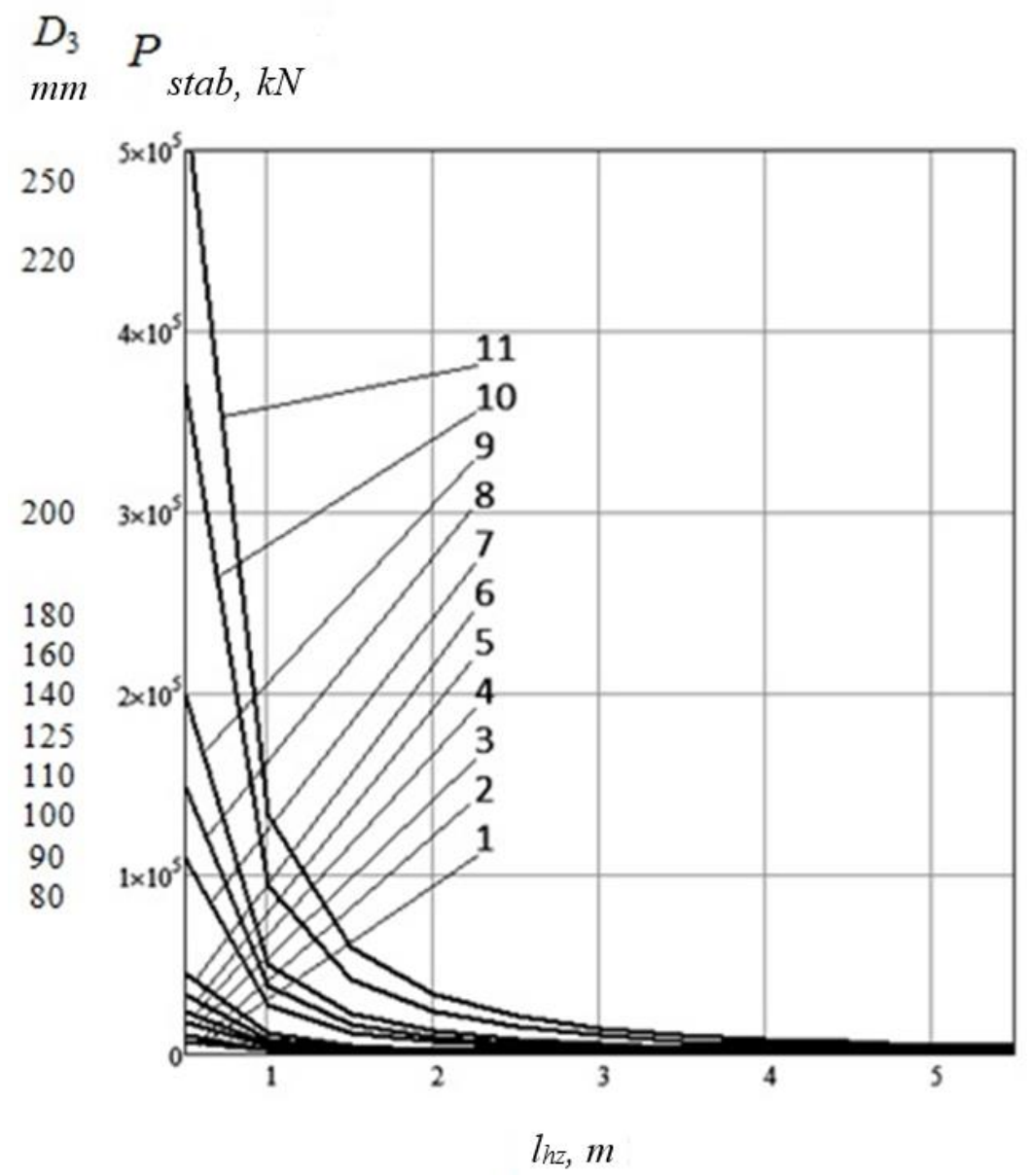

Fig. 4. The dependences of the force $P_{S_{\text {stab }}}$ (curves $1 \ldots 11$ ) on the total length of the hydraulic cylinder lhz for various values of the inner diameter of the liner D3 $=80 \ldots 250 \mathrm{~mm}$, respectively.

In Figures 5 and 6, the condition $P_{S_{\mathrm{hz}}}=P_{S_{\mathrm{sab}}}$ is displayed by the intersection of the curve $P_{S_{\text {stab }}}$ corresponding to the size $D_{3}$ with a similar straight line $P_{S_{h z}}$ with the mark of the length of the hydraulic cylinder $l_{\text {hz }}$.It can be seen that with an increase in its diametric dimensions, increasing the rigidity of the hydraulic cylinder, the likelihood of the loss of stability decreases as its length grows, as evidenced by the curve $D_{3}=160 \mathrm{~mm}$ in Figure 6 and all the curves $D_{3}=180 \ldots 250 \mathrm{~mm}$ in Fig. 7 . 
$\lambda$

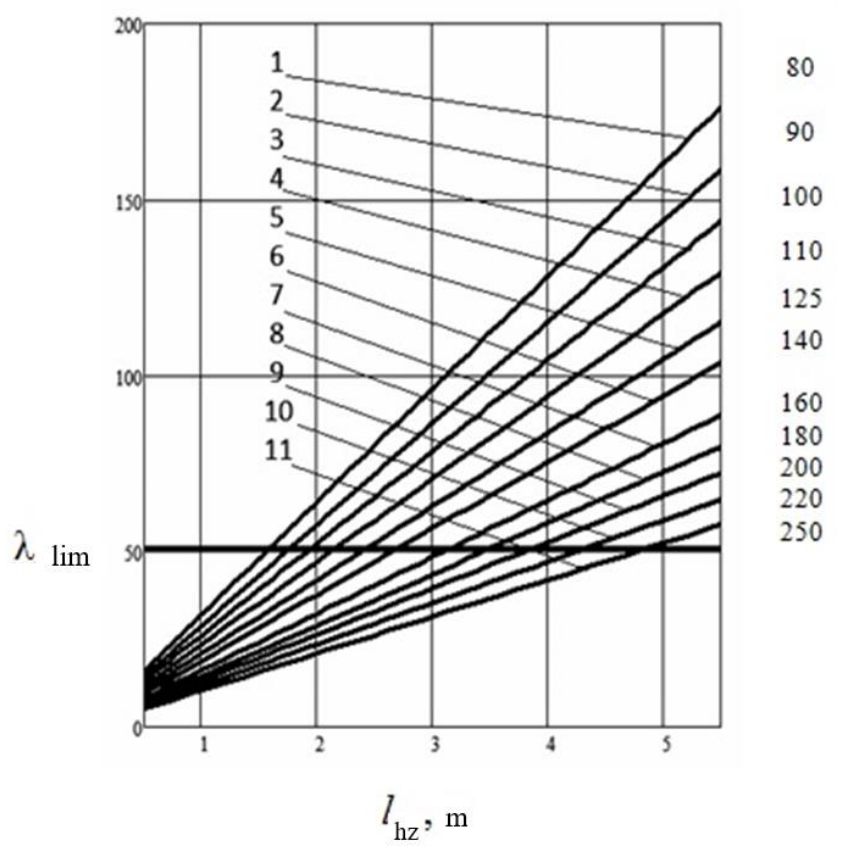

Fig.5. The relationship of the force $P_{S_{\text {sab }}}$ (curves $1 \ldots 4$ ) and the load $P_{S_{\mathrm{hc}}}$ on the rod (lines $5 \ldots 8$ ) with the full length of the hydraulic cylinder lhe for the values of the inner diameter of the liner D3 $=80$ and $90 \mathrm{~mm}$ at the pressure $\mathrm{p}=16 \mathrm{MPa}$ and D3 $=100$ and $110 \mathrm{~mm}$ at the pressure $\mathrm{p}=20 \mathrm{MPa}$.

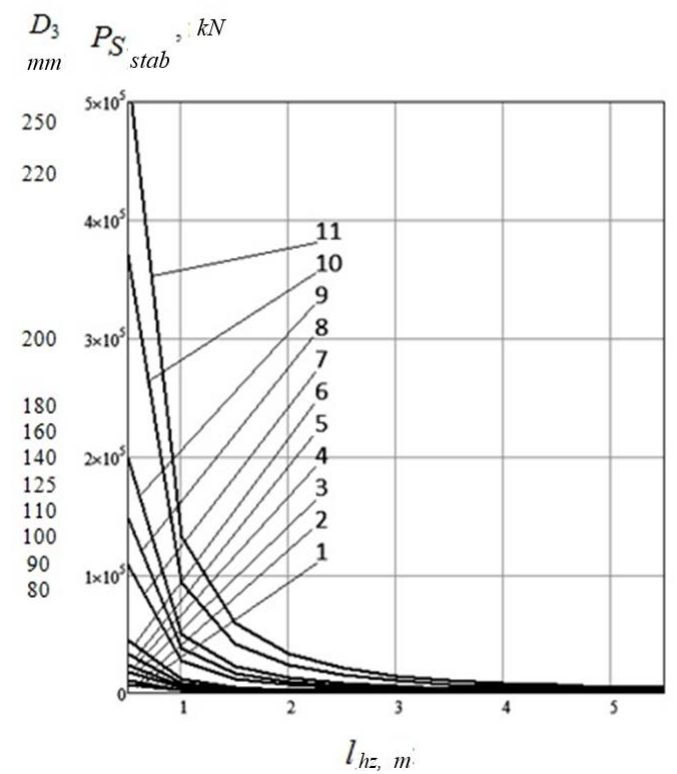

Fig.6.The relationship of the force $P_{S_{\mathrm{sab}}}$ (curves $1 \ldots 3$ ) and the force $P_{S_{\mathrm{hc}}}$ on the rod (lines $4 \ldots 6$ ) with the full length of the hydraulic cylinder lhe for the values of the inner diameter of the liner D3 $=125$ $\mathrm{mm}$ at the pressure $\mathrm{p}=20 \mathrm{MPa}$ and D3 $=140$ and $160 \mathrm{~mm}$ at the pressure $\mathrm{p}=25 \mathrm{MPa}$. 


\section{$\lambda$}

\section{$D_{3}$}

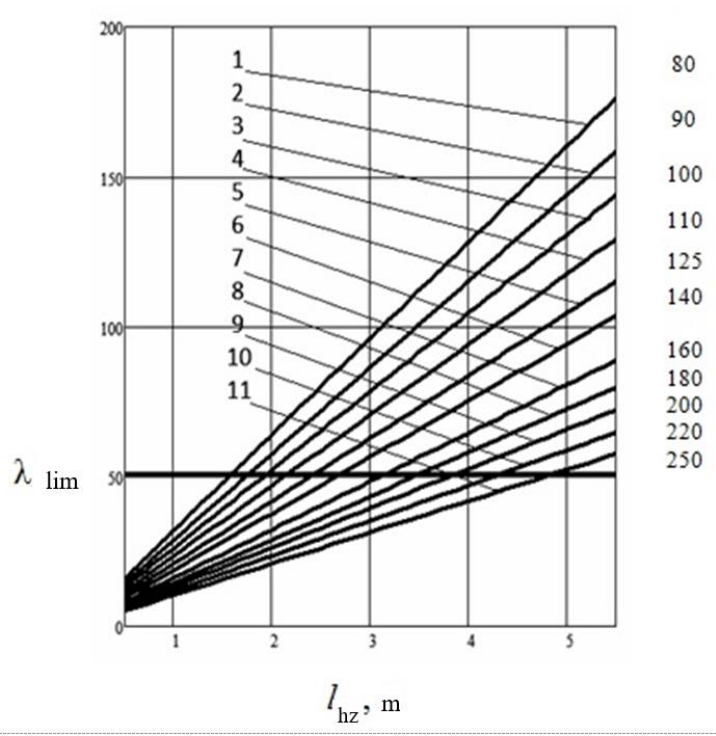

Fig.7. The relationship between the force $P_{S_{\text {stab }}}$ (curves $1 \ldots 4$ ) and the force $P_{S_{\mathrm{hc}}}$ on the rod (lines $5 \ldots$ 8 ) with the full length of the hydraulic cylinder lhz for the inner diameter of the liner D3 $=180,200$, 220 and $250 \mathrm{~mm}$ at the pressure $\mathrm{p}=25 \mathrm{MPa}$.

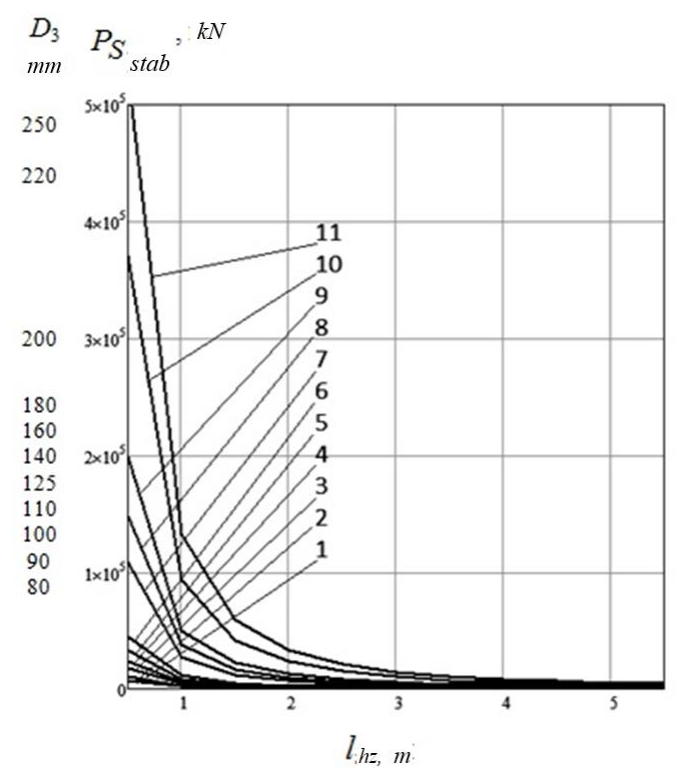

Fig.8. The dependences of the total length of the hydraulic cylinder lhz (lines $1 \ldots 11$ ) on the pressure $\mathrm{p}=16 \ldots 20 \ldots 25 \mathrm{MPa}$ in its piston cavity for various values of the inner diameter of the liner D3 = $80 \ldots 250 \mathrm{~mm}$, respectively.

Generalized graphical information of Figures 5-7, obtained due to the transformation of condition (14) with respect to the full length of the hydraulic cylinder $l_{\mathrm{hc}}$ 


$$
l_{\mathrm{hz}}=\sqrt{\xi \frac{\pi^{2} E_{1}\left(D_{5}^{4}-D_{3}^{4}\right)}{16 D_{3}^{2} p}}
$$

and the information presented in Figure 8 makes it possible to estimate the greatest possible length $l_{\text {hc }}$ of the designed hydraulic cylinder at a given pressure $p$ of the working fluid in the piston cavity for various values of the inner diameter of its liner $D_{3}=80 \ldots 250$ $\mathrm{mm}$.

In this case, it is mandatory to check the operability of the designed hydraulic cylinder using the stability criterion proposed above (12), the result of which can be the following possible combinations of loads $\left[P_{S_{\text {bend }}}^{\text {hor }}\right],\left[P_{S_{\text {bend }}}^{\text {vert }}\right]$ and $\left[P_{S_{\text {stab }}}\right]$ by their values:

$$
\begin{aligned}
& {\left[P_{S_{\text {bend }}}^{\text {hor }}\right]>\left[P_{S_{\text {stab }}}\right]<\left[P_{S_{\text {bend }}}^{\mathrm{vert}}\right] \text {; }} \\
& {\left[P_{S_{\text {bend }}}^{\text {hor }}\right]<\left[P_{S_{\text {stab }}}\right]<\left[P_{S_{\text {bend }}}^{\text {vert }}\right] \text {; }} \\
& {\left[P_{S_{\text {bend }}}^{\text {hor }}\right]<\left[P_{S_{\text {stab }}}\right]>\left[P_{S_{\text {bend }}^{\text {vert }}}^{\text {val }}\right]}
\end{aligned}
$$

taking into account that the condition $\left[P_{S_{\text {bend }}}^{\text {hor }}\right]<\left[P_{S_{\text {bend }}}^{\text {vert }}\right]$ is always true, as follows from the analysis of records (6) and (7),.

Here, the first inequality (16) corresponds to the condition of disruption of the hydraulic cylinder's operability due to the possible loss of its bearing capacity [5] as a result of loss of stability, while the second (17) and third (18) entries indicate the same, but because of the extreme longitudinal-transverse deformation of the long bearing elements of the hydraulic cylinder, mainly its rod (Fig. 1).

It should be noted that hydraulic cylinders with great flexibility must first be tested for stability. For this, it is recommended to use the proposed stability criterion (12). Moreover, in all cases, one should take into account the parameters of their greatest operational loading and the characteristics of the spatial arrangement, as applied to a specific RCM.

If the condition $\lambda<\lambda_{\text {lim }}$ or the last two combinations of forces $\left[P_{S_{\text {bend }}^{\text {hor }}}^{\text {hend }}\right],\left[P_{S_{\text {bend }}}^{\text {vert }}\right]$ and $\left[P_{S_{\text {stab }}}\right]$ are present, the hydraulic cylinder operability should be assessed by the characteristics of the stress-strain state of the long elements of the hydraulic cylinder and the reactions in its movable, sealed "piston - liner" and "rod - guide sleeve" couplings.

The main drawback of the existing design of a double-acting reciprocating hydraulic cylinder with a one-sided rod is that before the application of the operational longitudinal compressive force, it has full deflection, defined as the sum of the deflection due to the misalignment of its main load-bearing elements (rod and liner), because of the presence of gaps in its "piston - liner" and "rod - guide sleeve" couplings, deflection as a result of the presence of a possible initial (technological) curvature of the long elements (rod and body), regulated by the technological tolerance of misalignment of manufacturing long products, as well as the deflection from the action of shear forces - the weights of these elements (Figure 9 and 10.). After the application of the operational longitudinal compressive force (Fig. 11), that is, when liquid is supplied under pressure to the piston cavity of the hydraulic cylinder, the total deformation of the hydraulic cylinder (Fig. 12) increases [1] and, being the shoulder of the application of this force, leads to an increase in the total bending moment, which can cause critical stresses and, accordingly, the appearance of plastic deformations at the hydraulic cylinder rod and the subsequent loss of operability by the 
hydraulic cylinder as a result of jamming of the rod with the piston in the hydraulic cylinder body (liner).

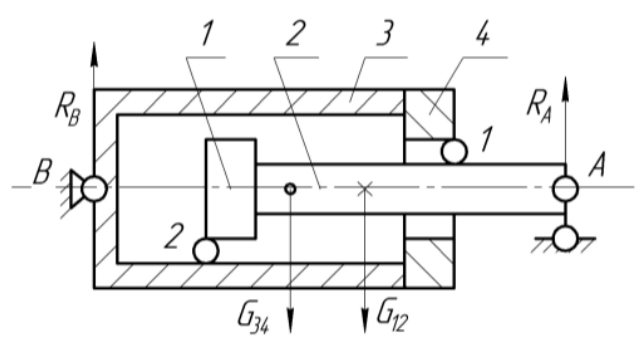

Fig.9. The structural and design diagram of hydraulic cylinder loading: 1 - the piston; 2 - the rod; 3 the liner (case); 4 - the guide sleeve (axle box).

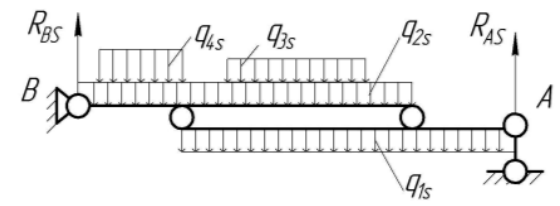

Fig.8. The dependences of the total length of the hydraulic cylinder lhz (lines $1 \ldots 11$ ) on the pressure $\mathrm{p}=16 \ldots 20 \ldots 25 \mathrm{MPa}$ in its piston cavity for various values of the inner diameter of the liner D3 = $80 \ldots 250 \mathrm{~mm}$, respectively.

As the friction surfaces of the elements of the hydraulic cylinder wear out, again leading to an increase in its total deformation (Fig. 13), respectively, to an increase in the acting longitudinal-transverse loads, stresses (Fig. 13) and reactions (Fig. 14) in movable pressurized couplings, the operating conditions of the hydraulic cylinder deteriorate with greater intensity [5], which leads to a decrease in its reliability and service life, both in its bearing and in sealing capacities [6].

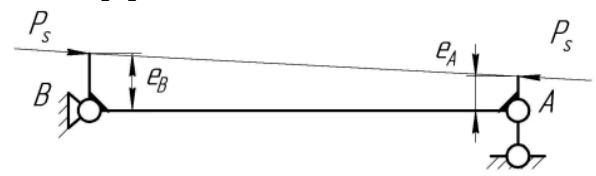

Fig.11.The design diagram of the eccentric loading of the hydraulic cylinder with longitudinal compressive force PS.

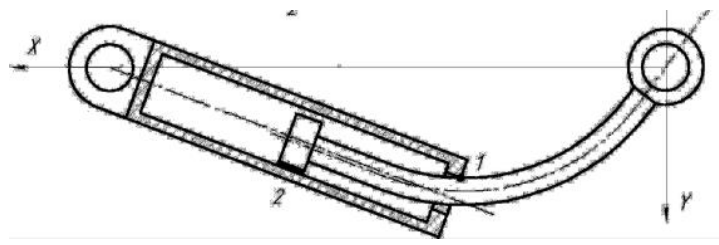

Fig.12. The schemes of operational deformation of the hydraulic cylinder and its elements. 


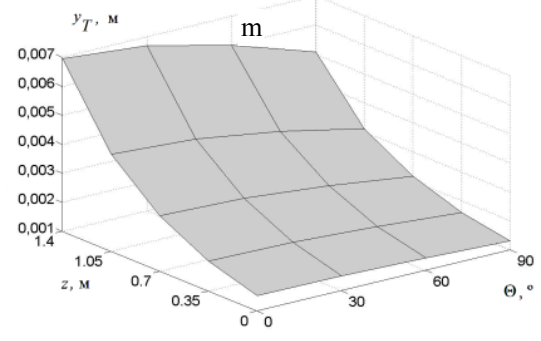

$\mathrm{m}$

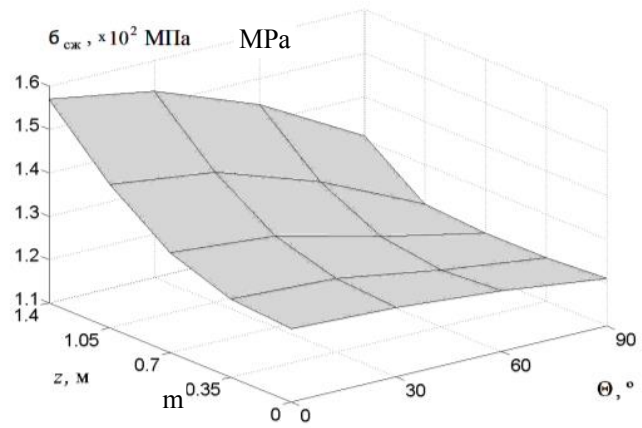

Fig.13.The complete deflection y $\gamma$ and compression stress $\sigma \mathrm{COMP}$ in the dangerous section of the traditional hydraulic cylinder rod.
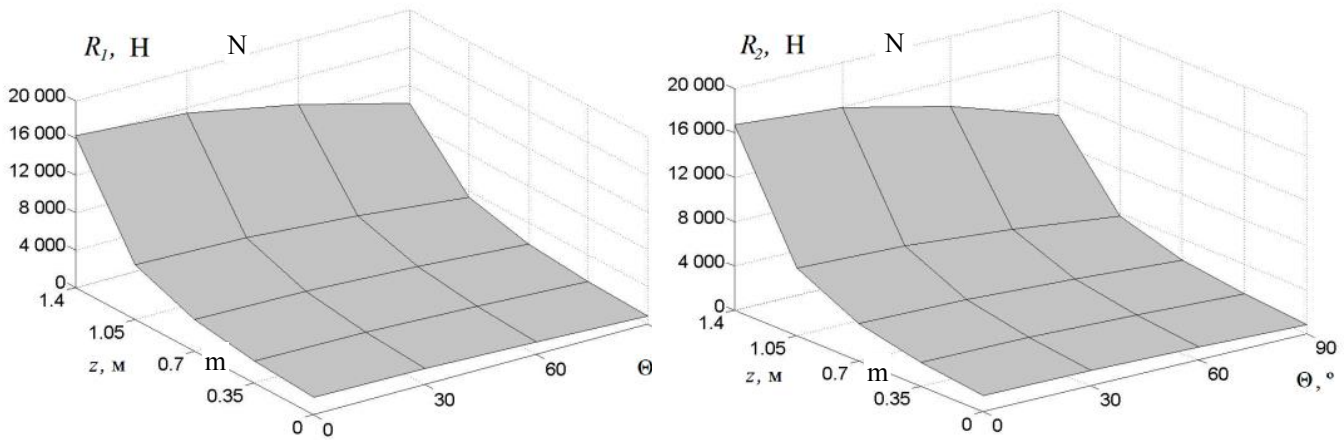

Fig. 14. The dependences of the reactions R1 and R2 in movable hermetic couplings "rod - guide sleeve" and "piston - liner" of a conventional hydraulic cylinder from the angle $\Theta$ of its inclination to the horizon and the position $\mathrm{z}$ of the rod.

\section{Discussion}

The listed disadvantages of the traditional hydraulic cylinder design can be eliminated by bringing the hydraulic cylinder from the state of longitudinal-transverse bending to the state of stability or close to that through the support of the hydraulic cylinder body (liner) with an intermediate sensor support (Fig. 15).
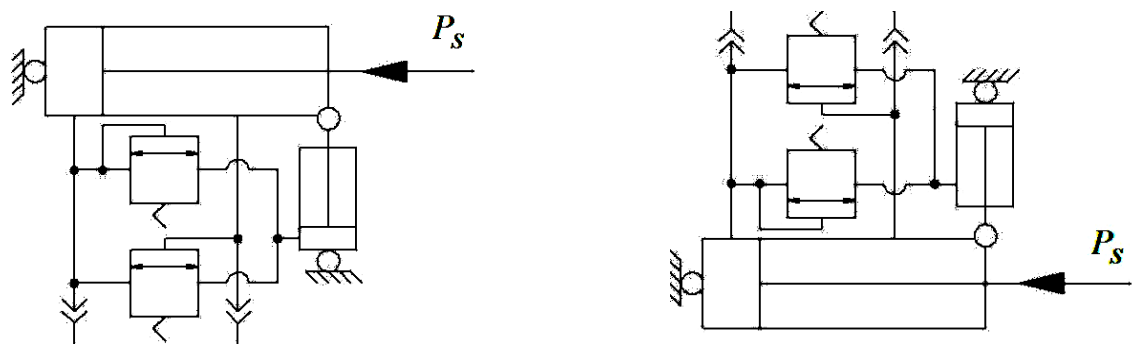

Fig.15. The schematic diagram of the sensor support of the hydraulic cylinder according to a.s. USSR No. 1386758 and No. 1735620, according to Patents of the Russian Federation No. 2046893 and No. 2050479. 
Bringing the hydraulic cylinder from the state of longitudinal-transverse bending to the state of stability or close to that through supporting the body (liner) of the hydraulic cylinder by an intermediate sensor backup support suggests several possible support options depending on the amount of supporting force when the following conditions are met, namely: the absence of reaction in the movable sealed "rod - guide sleeve" coupling, that is, $R_{1}=0$ (Fig. 16); the absence of reaction in the movable sealed "piston - liner" coupling, that is, $R_{2}=0$ (Fig. 17) and the absence of a complete deflection of the hydraulic cylinder at the point of connection of the hydraulic cylinder body with the backup support, that is, $y_{T}=0$ (Fig. 18), which is practically accompanied by lack of full deflection of the hydraulic cylinder rod.

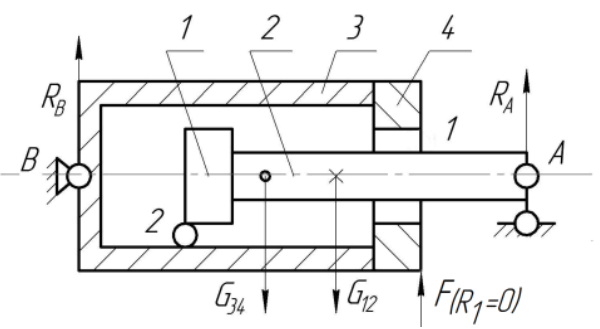

Fig.16. The schematic diagram of the support of the hydraulic cylinder with the force $F_{\left(R_{1}=0\right)}$.

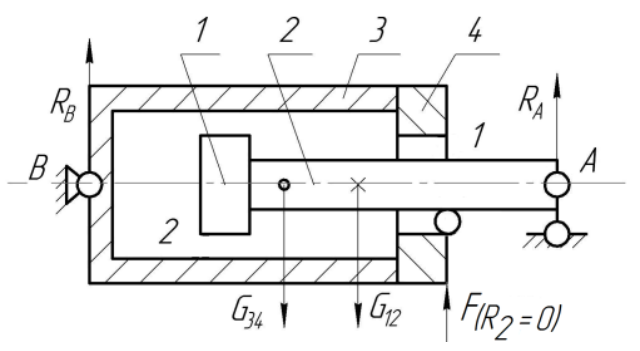

Fig.17. The schematic diagram of the support of the hydraulic cylinder with the force $F_{\left(R_{2}=0\right)}$.

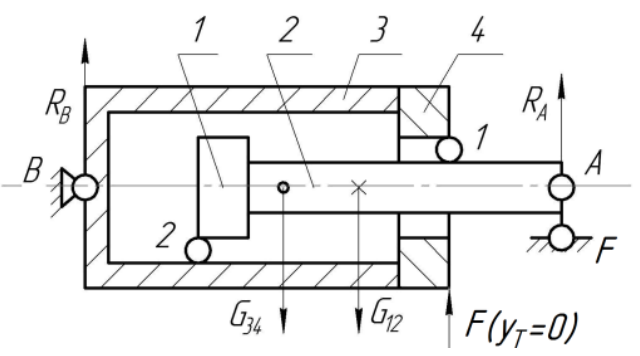

Fig.18. The schematic diagram of the support of the hydraulic cylinder with the force $F_{\left(y_{\gamma}=0\right)}$. 


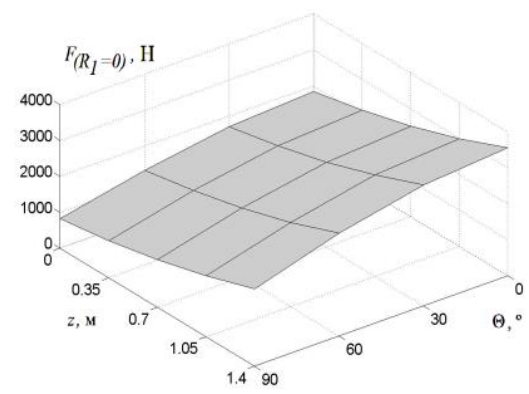

Fig.19. The support force $\mathrm{F}$ realized by the backup support for the condition $\mathrm{R} 1=0$.

For the comparative analysis of the characteristics of the traditional and promising hydraulic cylinders with different versions of the sensor intermediate support, the reaction values $R_{1}$ and $R_{2}$, as well as the total deflection $y_{\gamma}$ at the coupling point of the hydraulic cylinder body with the intermediate sensor backup support, which uses an auxiliary singleacting hydraulic cylinder with one-sided stock, are taken into account.

A joint analysis of the schemes (Fig. 19-21) and dependences (Fig. 8) show that the most preferred option for supporting the hydraulic cylinder is the condition under which the reaction is $R_{1}=0$, and the support force $F=\min$.

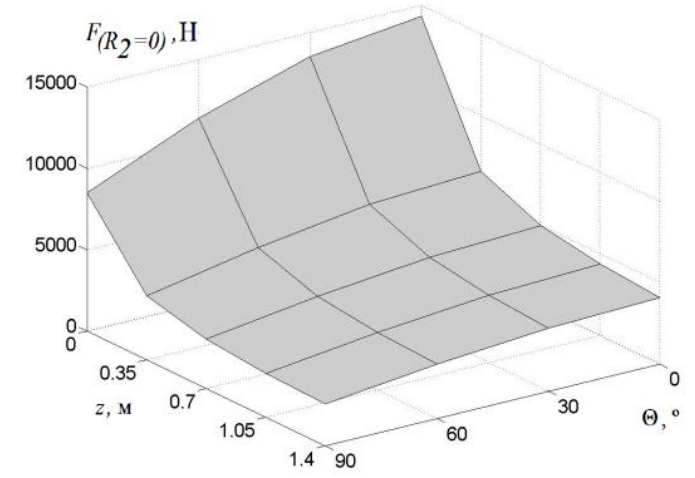

Fig. 20. The support force F realized by the backup support for the condition $R 2=0$.

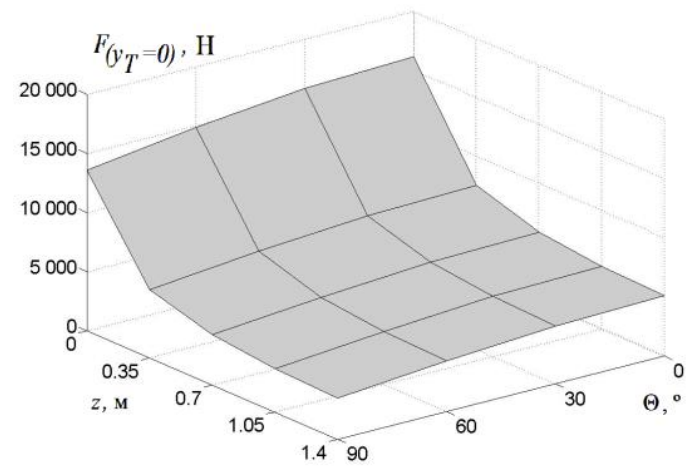

Fig. 21. The support force F realized by the backup support for the conditions $\mathrm{yT}=0$.

In addition, as follows from the analysis of the dependences shown in Figures 19-21, this support option is characterized by the minimum value of $R_{2}$ reaction in the "pistonliner" coupling (Fig. 22). 

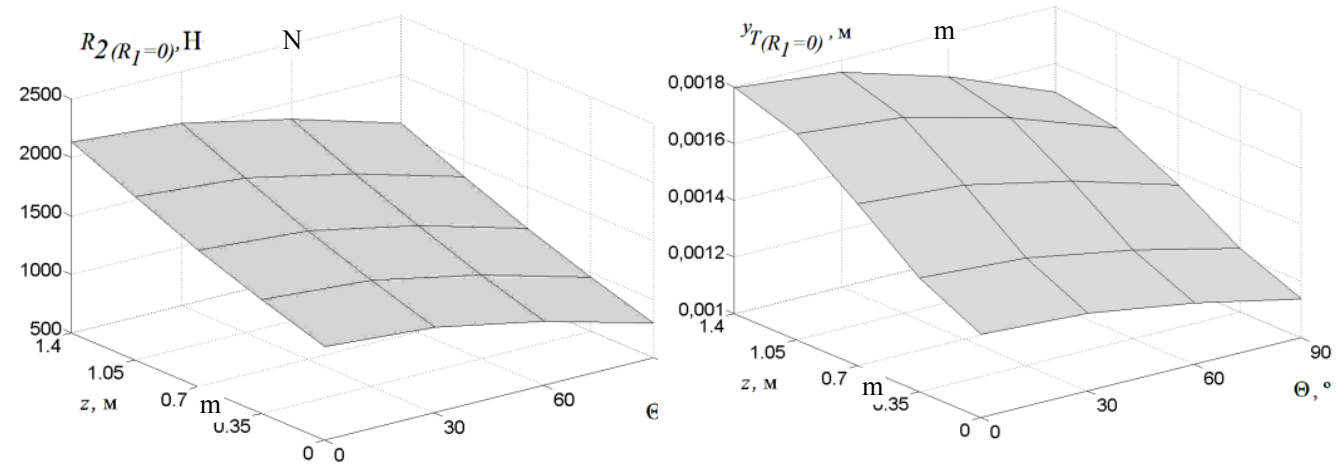

Fig.22. The reaction $R_{2}$ and deflection $y_{T}$ provided that support $R_{1}=0$.

Moreover, judging by the direction of the reactions (Fig. 23 and 24), with the support of the hydraulic cylinder, according to the last two options, the contact points of the long supporting elements of the hydraulic cylinder change to the opposite.
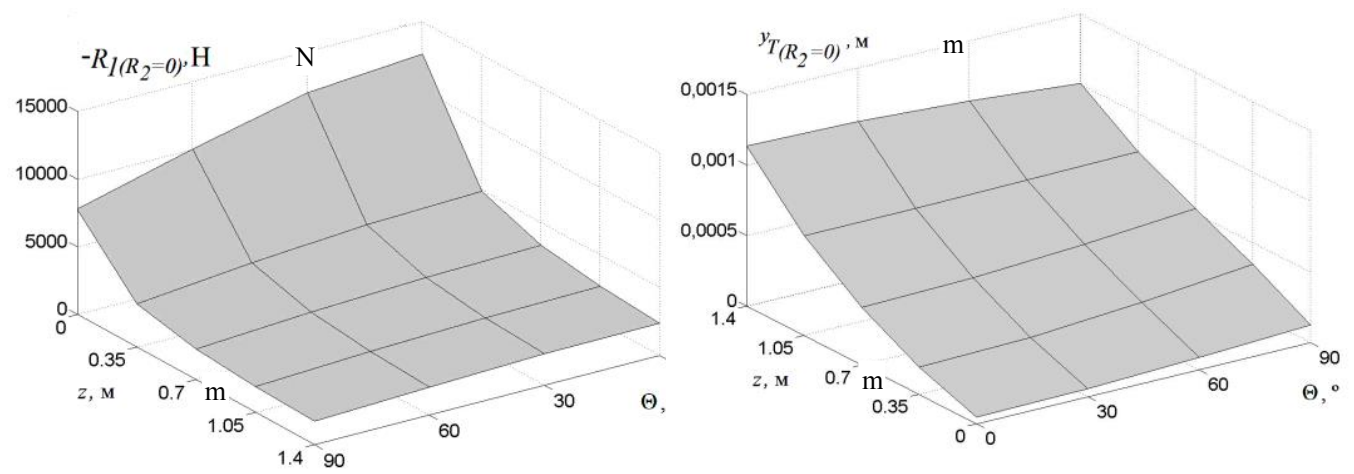

Fig.23. The reaction $R_{1}$ and deflection $y_{T}$, provided that support $R_{2}=0$.

Regarding the total deflection $y_{T}$, it should be noted that the second support option, in relation to the first one, is somewhat preferable (Figs. 23 and 24).
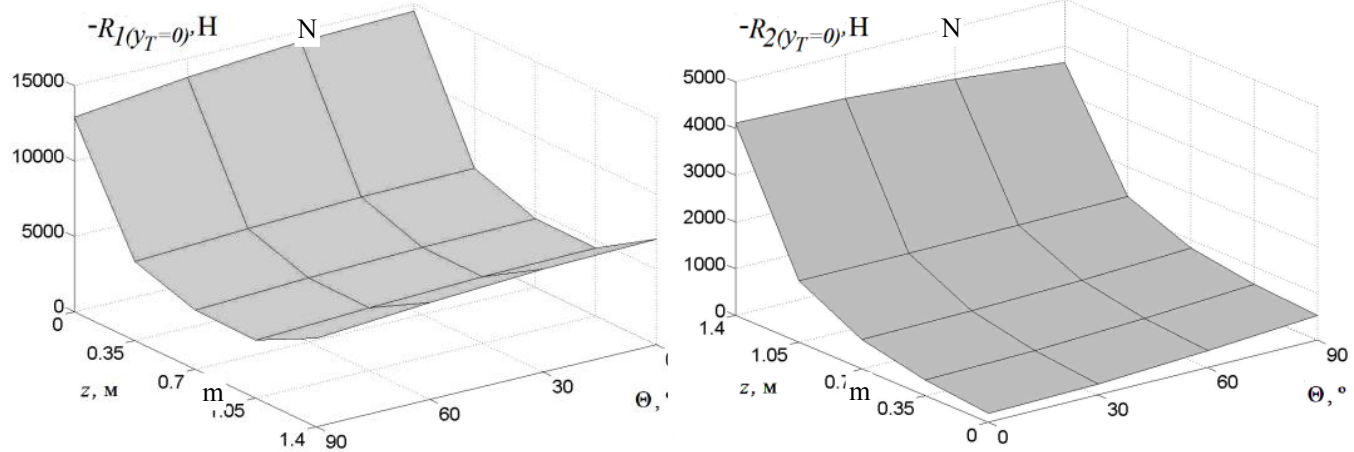

Fig. 24. The reactions $R_{1}$ and $R_{2}$ provided that support $y_{T}=0$. 
However, which is noteworthy, both values are much less than the total deflection of the hydraulic cylinder of the traditional design (Fig. 13), which confirms the relevance and feasibility of the research conducted in this direction.

\section{Conclusions}

1. The most preferred option for supporting the hydraulic cylinder should be considered the one with an intermediate sensor backup support, which realizes the support force $F_{\left(R_{1}=0\right)}$, additionally relieving the least reliable "rod - guide sleeve" coupling of the hydraulic cylinder, bordering on the environment saturated with abrasive dust.

2. Reducing the reactions $R_{1}$ to zero and $R_{2}$ by more than seven times acting in movable sealed "rod - guide sleeve" and "piston - liner" couplings, which, in turn, contributes to a significant reduction in the wear rate of their constituent elements, which increases the durability of the hydraulic cylinder.

3. A decrease of the total deflection $y_{\gamma}$ more than four times is accompanied by a decrease in stresses $\sigma_{\text {COMP }}$ by $(13 \ldots 15) \%$.

4. In addition, the support of the hydraulic cylinder makes it possible to reduce the stress $\sigma_{\text {COMP }}$ due to the total decrease in the second and third summands of equation (1) by up to $(10 \ldots 20) \%$.

5. When creating an intermediate sensor backup support, it is necessary to take into account the nonlinear nature of the support force $F$.

6. The design parameters of the auxiliary hydraulic cylinder of the intermediate sensor support should be considered for a specific hydroficated RCM taking into account its working process and loading mode. So, for example, the working process of the hydraulic cylinder of the handle of the bucket excavator of size IV with the backhoe is limited by the characteristics $z$ and $\Theta$, respectively $(0.0 \mathrm{~m} \ldots 0.8 \mathrm{~m})$ by $\left(17^{\circ} \ldots 18.5^{\circ}\right)$, which greatly simplifies the design of the supports.

7. All of the above indicates the relevance of research in this direction, the results of which contribute to increasing the efficiency of the use of hydroficated RCM in general.

\section{References}

1. D.Yu. Kobzov, V.V. Zhmurov, I.O.,Kobzova, D. J.Lkhanag, J. Mod. Sc. 4, 28, 64-69 (2010)

2. A.G. Dibir, O.V. Makarov, N.I. Pekelny (Kharkov: KhAI, 2008)

3. M.D. Worley, V. LaSaponara Proc. IMECE- 2007 (2007)

4. J.D. Zimmerman, M.Pelosi, .A.Williamson Proc. IMECE-2007 (2007)

5. D.Yu.Kobzov, V.V.Zhmurov, I.O.Kobzova, V.G.Gubanov J. Mod. Sc. 2, 50, 49-58 (2016)

6. V.A. Marutov, S.A. Pavlovsky (Moscow, Mashinostroenie, 1966)

7. V. Goremikins, K. Rocens, D. Serdjuks, L. Pakrastins, N. Vatin. Advances in Civil Engineering and Building Materials IV - Selected and Peer Reviewed Papers from the 2014 4th International Conference on Civil Engineering and Building Materials, CEBM 2014. 363-368 (2015)

8. V. Goremikins, D. Serdjuks, K. Buka-Vaivade, L. Pakrastins, N. Vatin. Baltic Journal of Road and Bridge Engineering. 2017. 12(4). 234-240. DOI:10.3846/bjrbe.2017.29 